\title{
Peak Oxygen Consumption
}

National Cancer Institute

\section{Source}

National Cancer Institute. Peak Oxygen Consumption. NCI Thesaurus. Code C153553.

The highest rate of oxygen consumption a subject reaches during an aerobic capacity test. 